\title{
Therapeutics of the use of the Gum in Gum in its Different Presentations to Minimize the Inertia and Privacy of the Dream
}

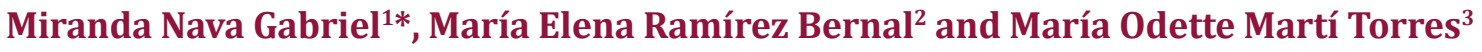 \\ ${ }^{1}$ Chief Neurologist of the Neurology Service of the Regional Military Hospital of Specialties of Guadalajara, Mexico \\ ${ }^{2}$ Internal Doctor of the Regional Military Specialties, Hospital of Guadalajara, Mexico \\ ${ }^{3}$ Dentist and Specialist in Orthodontics and Maxillary Orthopedics, Autonomous Mexico State University, Mexico
}

*Corresponding author: Miranda Nava Gabriel, Chief Neurologist of the Neurology Service of the Regional Military Hospital of Specialties of Guadalajara, Mexico

\section{ARTICLE INFO}

Received: 幽 November 11, 2019

Published: 幽 November 25, 2019

Citation: Miranda Nava Gabriel, María Elena Ramírez Bernal, María Odette Martí Torres. Therapeutics of the use of the Gum in Gum in its Different Presentations to Minimize the Inertia and Privacy of the Dream. Biomed J Sci \& Tech Res 23(2)2019. BJSTR. MS.ID.003865.

\section{ABSTRACT}

Introduction: Sleep is defined as the natural, periodic and reversible decrease in the perception of the external environment, with the preservation of a certain degree of reactivity to the environment and autonomous functions. The dream is composed of 2 phases, REM phase and NREM phase, these phases alternate during the night in the form of five to six cycles; of which the NREM phase is presented in $75 \%$ and the REM in $25 \%$.

Theoretical Framework: Caffeine is a readily available short-acting stimulant that has been shown to reduce some of the deficits associated with sleep loss. With gum marking absorption is carried out on the oral mucosa, which generates a greater bioavailability of the active substance and an immediate mechanism of action. The effective response dose can range from $100 \mathrm{mg}$ to $200 \mathrm{mg}$ and the effect arises from 6 min after the active substance is administered. It is important to take into account that the dose of caffeine in chewing gum is directly proportional to the effects on sleep inertia, as well as the duration and maintenance thereof.

Discussion: Being able to analyse the mechanisms of action of caffeine on sleep inertia, helps us to make a comparison of gum with caffeine vs placebo (simple chewing gum). Chewing generates for a short period the maintenance of performance on simple and complex tasks and improves alertness, with caffeine these same effects are prolonged for longer.

Conclusion: Gum with caffeine is an effective therapeutic presentation on sleep inertia. The dose to obtain a positive response ranges from 75 to $200 \mathrm{mg}$ depending on the circumstances of the sleep restriction. That is, thanks to the effects generated by chewing on the state of alertness and cognition and their potentialization and duration by caffeine.

\section{Introduction}

Sleep disorders cause alterations in the quality of life of each and every patient. At least two thirds of those suffering from chronic degenerative diseases are affected by sleep disorders, the most frequent being the female sex. Many individuals are subjected to night work where a constant alertness is required and it has been scientifically proven how this affects the quality of life of each employee; Well, sleep is one of the most essential pillars of daily life, since it maintains critical aspects of cognition for optimal mental performance, as well as in mood, alertness and performance during the work and activities of everyday life. Waking up abruptly implies 
something known as "sleep inertia," which is characterized by impaired performance and subjective lack of alert for a transitional period, which hardly progresses to good cognition.

It is well known that chewing facilitates concentration, maintains alertness and improves performance in cognitive tasks, but the question is how effective is the effect of chewing a simple chewing gum on its own vs. a containing caffeine? Therefore, one of the objectives of this research work is to analyze the effects of chewing a simple chewing gum to one that contains caffeine, verify the effectiveness by reversing the inertia of each individual's sleep and know the mechanism of action, pharmacokinetics, bioavailability and absorption of a gum with caffeine during sleep deprivation.

\section{Theoretical Framework}

Normal sleep progresses in various stages: NREM phase (nonrapid eye movement) and REM phase (rapid eye movement). These cycles alternate overnight in the form of 5 to 6 cycles. In $75 \%$ of normal nighttime sleep it is NREM and in 25\% REM.

\section{REM Phase}

It happens every 90 minutes approximately. It is characterized by null muscle tone, presence of active sleep, since the cerebral electrical activity is maximum while the body is completely at rest, rapid eye movements, heart rate as well as respiratory show irregular signs, evidence of increased metabolism Basal and the amount of gastric juice [1].

\section{NREM Phase}

Also known as "deep sleep," it facilitates body rest and consists of 3 phases.

A. Phase N1 (Superficial Sleep): very light sleep stage, lasting several minutes. It is characterized by a slight decrease in heart rate, breathing, muscle tone, general state of deep rest, relaxed and sleepy, keeping active the ability to perceive external stimuli.

B. Phase N2 (superficial sleep): it is characterized in the EEG by sleep spindles and complexes $\mathrm{k}$. Its duration is 10 to 15 minutes. In this phase the muscle tone relaxes even more, slightly decreases body temperature and respiratory and heart rate, disappearing eye movements.

C. Phase N3 (deep sleep): it is characterized by a global slowing of the electrical path and the appearance of slow waves and high voltage (deltas) whose total duration must be greater than $20 \%$ and less than $50 \%$ of the path.

Sensory perception decreases markedly, as do heart and respiratory rates. The relaxation of the muscles intensifies. It is more difficult to wake up the subject, and if he does, he is disoriented and confused. It is the fundamental stage for the subject to rest subjectively and objectively. This research work was carried out based on various medical studies, where it is approached from the speed of absorption, bioavailability, the pharmacokinetics of caffeine in chewing gum, the effects of chewing, how caffeine reverses sleep inertia, assess cognitive performance, mood and alertness after administering caffeine gum until the composition, formulation and design of said studied chewing gum.

In addition, each and every one of the selected articles will be included in a systematized way to carry out a bibliographic review and thus carry out a complete study of the effects and efficacy of caffeine gum to minimize sleep inertia, based on the scientific evidence of some experimental studies in which there is certainty of its efficacy, since caffeine is involved in the autonomic nervous system and exerts its effects by acting as an adenosine receptor antagonist.

The approach is an essential part in the daily life of the human being, since it is the pillar to be able to carry out any cognitive activity, maintain an effective and constant performance in order to successfully complete actions of daily life and the days of the job. Fatigue can cause various complications and over time can affect the health of the individual, also leads to a reduction in efficiency during the day and an increase in the incidence of any type of accident [2].

Caffeine is a white, odourless powder that can have different molecular presentations, it can range from an anhydrous substance to a water molecule. Caffeine is a methylxanthine that inhibits the phosphodiesterase enzyme, generating an antagonistic effect on central adenosine receptors, this adenosine is produced during daily activities and binds to its receptors, generating a feeling of fatigue and consequently a sleep induction. Due to the similarity of adenosine with caffeine, the latter takes place in the adenosine receptors and thus prevents the transmission of the fatigue signal generating that the person can continue performing their daily and work activities for a period of time. longer, because it creates a feeling of insomnia [3].

Caffeine is a central nervous system (CNS) stimulant that can promote wakefulness and increases mental activity. In addition, it can stimulate the respiratory center, increase the frequency and depth of breathing and increase total muscle work. Typically, caffeine is commonly consumed and / or administered as a liquid substance, although there are other types of presentations such as tablets or capsules. A new novel way to consume caffeine is through chewing gum which in turn can provide additional advantages, some of them are:

a) Absorption is carried out through the oral mucosa, resulting in a greater bioavailability of the active substance at systemic level.

b) Effective and immediate mechanism of action (oral mucosa). 
c) It is easy to use and is suitable even for pediatric patients or patients with difficulty swallowing tablets or tablets.

d) It generates less side effects since the active substance is released proportionally to chewing.

e) The primary hepatic metabolism of the active substance is avoided, as they are absorbed directly by the oral mucosa.

f) Lower risk of overdose due to chewing effect.

Chewing is a physiological motor activity that involves many neuronal pathways, this action is associated with increased blood flow at the cerebral and orofacial level which in turn implies efficacy to increase alertness, physical well-being and improve memory performance.

The active substance (caffeine) of chewing gum is released proportionally to chewing, this in turn is absorbed through the oral mucosa and another percentage of it is swallowed as a bolus with saliva, reaches the gastrointestinal tract and Because caffeine is soluble, the absorption rate is faster compared to that of the tablet. In addition, it is important to mention that the control of caffeine release in chewing gum it is for a prolonged time and improves the variability of the release and retention times of the drug, these other advantages of this new form being caffeine administration.

It is important to take into account that the effectiveness of this chewing gum with the active substance that is caffeine, has to be argued based on studies that corroborate this effectiveness, such is the case of the P300 signal that is obtained thanks to an electroencephalogram, this signal is a neural record that is projected as a positive deflection and in turn measures the potential of presence, magnitude, topography and duration of cognitive function signalling. The signal is acquired more strongly around the parietal electrodes, although it has been suggested that there are also interactions between the frontal and temporal regions. Recent studies comment that this $\mathrm{P} 300$ wave is composed of 2 secondary waves known as "P3A and P3B signals", these components respond individually to different stimuli and it has been suggested that the P3A wave originates in the frontal attention mechanisms directed by stimuli during task processing, while P3B originates from parieto-temporal activity associated with attention and memory processing. The potential related to the P300 signal had a shortened latency after the chewing gum, and the frontal and temporal beta power was increased by chewing the chewing gum after performing a sustained attention task. The quantitative effects on the EEG of chewing gum without cognitive performance appear to be moderated by taste, suggesting that chewing gum may alter the alertness in the absence of cognitive performance. Working under pressure, in other words, was associated with increased activity in the anterior cingulate cortex and the left frontal gyrus, where the motor neuronal regions of alertness and executive tasks are found. This same effect was found when chewing a chewing gum without taste or smell, which suggests that the motor activity of chewing may be a key factor in explaining these results, however it is not clear whether a higher level of motor activity in chewing will increase the associated effects, since there is evidence that more vigorous chewing or greater resistance to chewing does not moderate the effects on memory, the fact that chewing gum can increase arousal, therefore reaches a maximum peak and decreases and in turn decreases cognitive function in performing tasks that require attention (increase heart rate and beta power during monitoring), This suggests that it is more plausible that more vigorous chewing may have a greater effect on attention in the short term than on memory.

That is, chewing alone generates a potentialization of the performance of a simple task as a result of a reflex motor activity that is generated in the body in the face of sleep deprivation, but this does not mean that this performance is maintained. A study shows that administering only $200 \mathrm{mg}$ of caffeine in conjunction with chewing gum, not only improves the performance of simple and complex activities and / or tasks, but also improves alertness compared to chewing without the active substance. In turn, the period of pre-injection and autonomous cardiac activity remain unchanged during chewing with or without caffeine, generating a response reflected in the increase in parasympathetic activity with changes at the level of RR intervals in the EEG, the latter mentioned above. they are predictors of speed and accuracy in the most complex cognitive tasks during sleep deprivation.

the alert status and performance maintenance during the performance thereof. Some other studies show as evidence that the use of caffeine in chewing gum is also equally effective in sleep deprivation before a post-party; In a double-blind study with 15 adults as participants, they were given this chewing gum that contained $100 \mathrm{mg}$ of caffeine at the hour and at 6 hours after waking up vs the placebo, subsequently they were assigned psychomotor tasks before a surveillance at 0, 6, 12 and 18 minutes. The qualifier of the tests was carried out based on the speed of response and the number of hits during the tests. Within the results are observed that $100 \mathrm{mg}$ of caffeine did not completely restore performance, but showed an improvement in response speed by $85 \%$ compared to the $73 \%$ placebo; since the effect of caffeine was evident at 6 min after waking, which continued to improve performance until 18 minutes, that is, the results indicate that 100 $\mathrm{mg}$ of caffeine substantially attenuates the inertia of sleep before a sudden awakening by a valuable period of time, but at higher doses (200 mg), it is expected to more easily antagonize sleep inertia in its entirety for a longer period.

\section{Discussion}

The purpose of this article is to evaluate, based on a compilation of articles, the efficacy of caffeine as an active ingredient in a new therapeutic presentation vs placebo, studying the pharmacokinetics, bioavailability and half-life of caffeine. Within the bibliographic reviews that were addressed for the realization of this article, it is 
evident that caffeine in chewing gum is a new and new presentation that is effective in counteracting the inertia of sleep, improving alertness, cognition and maintaining performance during the simple and complex activities of daily life. It is important to take into account that the effects of caffeine are directly proportional to the dose administered in patients, as well as in the route of administration. The chewing of a chewing gum without the active substance (placebo) generates in a later way the maintenance of the performance as well as of the alert state with the difference that these effects appear during a very short period of time.

\section{Conclusion}

Caffeine is an odourless powder that inhibits the phosphodiesterase enzyme, which generates an antagonistic effect on the central adenosine receptors, resulting in antagonism of the transmission of the fatigue signal, promoting wakefulness and increased mental activity. The effect of chewing is associated with increased orofacial blood flow that increases alertness, physical well-being and memory performance. A combination of chewing gum with

\section{ISSN: 2574-1241}

DOI: 10.26717/BJSTR.2019.23.003865

Miranda Nava Gabriel. Biomed J Sci \& Tech Res

(c) (i) This work is licensed under Creative

Submission Link: https://biomedres.us/submit-manuscript.php caffeine potentiates and synergizes the effects that chewing alone generates, resulting in a new, novel and effective therapeutic presentation on sleep inertia, as it is easy to use and its absorption is performed through the oral mucosa, which generates a greater bioavailability of the active substance and a mechanism of immediate action, in turn these effects are present for a prolonged period since the caffeine inside the chewing gum is released so Proportional to chewing. Caffeine can provide better alertness and performance at doses of 75 to $150 \mathrm{mg}$ after acute sleep restriction and at doses of 200 to $600 \mathrm{mg}$ after one night or more of sleep loss. Caffeine is unlikely to have negative effects on sleep that follows 8 hours or more after administration. However, frequent use of caffeine can lead to tolerance and withdrawal syndrome.

\section{References}

1. Emsley J (2004) Vanity, vitality and virility: The science behind the products you love to buy. Oxford: Oxford University Press.

2. McKenzie D (1993) Chewing gum gave Stone Age punk a buzz.

3. Weaver V (2003) Wrigley patents antiimpotence gum.

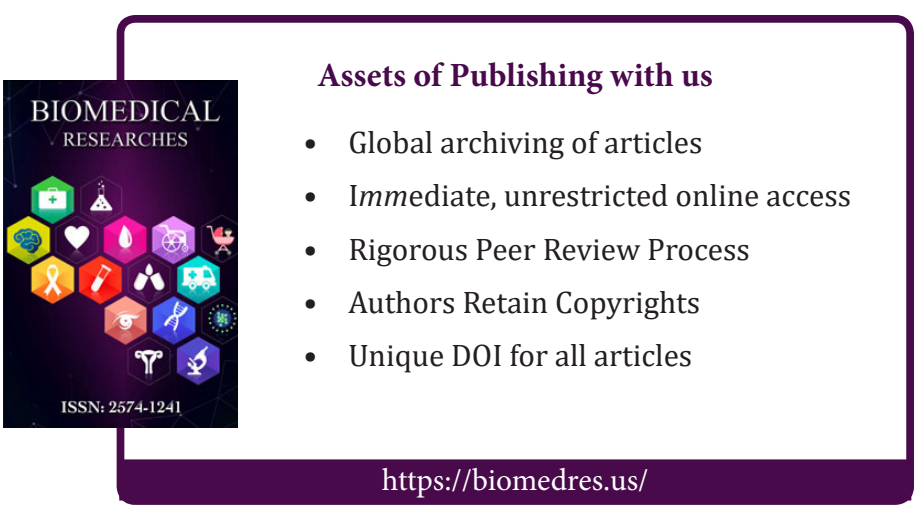

